\title{
Histone Acetyltransferase SIGCN5 Regulates Shoot Meristem and Flower Development in Solanum lycopersicum
}

\section{OPEN ACCESS}

Edited by:

Xigang Liu,

Hebei Normal University, China

Reviewed by:

Weibing Yang,

Institute of Plant Physiology and Ecology, Shanghai Institutes

for Biological Sciences, Chinese Academy of Sciences (CAS), China

Yun Zhou,

Purdue University, United States

*Correspondence:

Bo Sun

sunbo@nju.edu.cn

Specialty section:

This article was submitted to

Crop and Product Physiology,

a section of the journal

Frontiers in Plant Science

Received: 31 October 2021 Accepted: 21 December 2021

Published: 21 January 2022

Citation:

Hawar A, Xiong S, Yang Z and Sun $B$ (2022) Histone Acetyltransferase SIGCN5 Regulates

Shoot Meristem and Flower

Development in Solanum

lycopersicum

Front. Plant Sci. 12:805879.

doi: 10.3389/fpls.2021.805879

\section{Amangul Hawar, Shiqi Xiong, Zhen Yang and Bo Sun* \\ State Key Laboratory of Pharmaceutical Biotechnology, School of Life Sciences, Nanjing University, Nanjing, China}

The histone acetyltransferase (HAT) general control non-repressed protein 5 (GCN5) plays important roles in plant development via epigenetic regulation of its target genes. However, the role of GCN5 in tomato, especially in the regulation of tomato shoot meristem and flower development, has not been well-understood. In this study, we found that silencing of Solanum lycopersicum GCN5 (SIGCN5, Solyc10g045400.1.1) by virus-induced gene silencing (VIGS) and RNA interference (RNAi) resulted in the loss of shoot apical dominance, reduced shoot apical meristem (SAM) size, and dwarf and bushy plant phenotype. Besides, we occasionally observed extra carpelloid stamens and carpels fused with stamens at the late stages of flower development. Through gene expression analysis, we noticed that SIGCN5 could enhance SIWUS transcript levels in both SAM and floral meristem (FM). Similar to the known function of GCN5 in Arabidopsis, we demonstrated that SIGCN5 may form a HAT unit with S. Iycopersicum alteration/deficiency in activation 2a (SIADA2a) and SIADA2b proteins in tomato. Therefore, our results provide insights in the SIGCN5-mediated regulation of SAM maintenance and floral development in tomato.

Keywords: tomato, SAM, SIGCN5, SIWUS, SIADA2

\section{INTRODUCTION}

Plants have a unique ability to give rise to new organs continuously due to the indeterminate production of undifferentiated stem cells located in specific regions of meristems. The shoot apical meristem (SAM) gives rise to the aerial organs, and the maintenance of SAM is key for the development of plants and adaptation to the changes of external environment (Pfeiffer et al., 2017). Unlike Arabidopsis, tomato is a typical sympodial plant. After the formation of 8-10 leaves, tomato SAM terminated and transforms into inflorescence meristem (IM) and sympodial meristem (SYM), which are formed at the leaf axils beneath the IM to sustain continuous growth. Thereafter, IM transforms to floral meristem (FM) and initiates a second IM in the meantime (Schmitz and Theres, 1999; Périlleux et al., 2014). Tomato FMs generate four whorls of floral organs, namely, sepals, petals, stamens, and carpels, sequentially in concentric whorls (Sekhar and Sawhney, 1984).

In Arabidopsis, the maintenance of the stem cell pool in the SAM is regulated by CLAVATAWUSCHEL (CLV-WUS) feedback loop (Schoof et al., 2000). In this feedback loop, WUS could directly induce stem cell identity and the expression of the stem cell marker gene CLV3 (Yadav et al., 2011; Daum et al., 2014). The CLV genes including CLV1 and CLV3 repress WUS through signaling cascades (Shang et al., 2019; Han et al., 2020), therefore coordinating and balancing stem cell proliferation with differentiation. The $C L V$-WUS feedback loop appears to be highly conserved 
across different plant species (Somssich et al., 2016). In tomato, the mutation of SlCLV3 promotes stem cell overproliferation and results in extra floral organs and bigger fruits (Rodríguez-Leal et al., 2017). In SlWUS RNA interference (RNAi) lines, plants have reduced flower size and fruit locule numbers (Li et al., 2017). Changes in tomato meristem size have also been observed in fasciated (fas) and locule number (lc) mutants, both of which have misexpression of SlWUS and SlCLV3, respectively (Muños et al., 2011; Xu et al., 2015; Chu et al., 2019).

In various plant species, studies have discovered that the CLVWUS regulatory loop could be modified by many additional factors, which can contribute to plant growth and productivity (Galli and Gallavotti, 2016). Among these factors, histone modifications including acetylation or methylation on several lysine residues of $\mathrm{H} 3$ are important for gene expression during plant development (Servet et al., 2010). Histone acetyltransferases (HATs) can catalyze acetylation of specific lysine residues on histone $\mathrm{N}$-tails and leads to transcriptional regulation (Bannister and Kouzarides, 2011). It has been reported that in most cases, GCN5 acts as the catalytic core of the HAT complex, which also include vital adaptor proteins ADA2a and ADA2b (Shahbazian and Grunstein, 2007). GCN5 acetylates lysine 14 of histone $\mathrm{H} 3$ (H3K14ac) and influences H3K9ac and H3K27ac levels in promoter region of its targets (Benhamed et al., 2006; Servet et al., 2010; Ruggieri et al., 2020). In contrast, ADA2 proteins could help increase the HAT activity of GCN5 (Mao et al., 2006).

In Arabidopsis, both GCN5 and ADA2b are required for many developmental processes such as shoot apical dominance, root meristem activity, leaf development, IM or FM function, and flower fertility (Bertrand et al., 2003; Vlachonasios et al., 2003; Cohen et al., 2009; Kornet and Scheres, 2009; Anzola et al., 2010; Servet et al., 2010). In poplar trees, ABREmotif binding protein PtrAREB1-2 binds to PtrNAC genes, recruits the HAT unit ADA2b-GCN5 by forming a AREB1ADA2b-GCN5 protein complexes, and results in increased H3K9 acetylation levels on PtrNAC genes (Li et al., 2019). In rice, the homeodomain protein OsWOX11 recruits a HAT complex containing OsGCN5 to establish the programs of cell proliferation in crown root meristem (Zhou et al., 2017). One study implies that the SAGA (Spt-Ada-GCN5 acetyltransferase) complex is an evolutionarily conserved complex that has a critical role in various developmental processes (Spedale et al., 2012).

In this work, we identified SIGCN5, SIADA2a, and SIADA2b in tomato and found that SIGCN5 can form a HAT unit with SIADA2a and SIADA2b and influences H3K9ac, H3K14ac, and $\mathrm{H} 3 \mathrm{ac}$ at the genomic level. Silencing of SlGCN5 resulted in dwarf plant phenotype, reduced SAM size, carpelloid stamens, and fusion of carpels with stamens in flowers. Furthermore, we proposed that SIGCN5 could enhance SlWUS expression, thereby maintaining stem cell homeostasis in tomato.

\section{MATERIALS AND METHODS}

\section{Plant Materials and Growth Conditions}

Arabidopsis plants and wild-type (WT) tomato (Solanum lycopersicum) plants of Micro-Tom (MT) and transgenic
Arabidopsis and tomato lines were grown in the greenhouse, under long-day condition (16-h light/8-h dark). For transformation, tomato cotyledons were cultivated in vitro in MS medium in a growth chamber (Panasonic, MLR-352H-PC) at $22^{\circ} \mathrm{C} / 20^{\circ} \mathrm{C}$ under 16 -h light and 8 -h dark conditions.

\section{Construction of TRV-SIGCN5 and RNAi Vectors and Tomato Transformation}

The tobacco rattle virus (TRV)-based vectors, i.e., pTRV1 and pTRV2, were used for virus-induced gene silencing (VIGS). To construct a pTRV2-SlGCN5 vector, according to the website ${ }^{1}$, a 400-bp DNA fragment of the SlGCN5 CDS was amplified from tomato cDNA using primers in Supplementary Table 1. The constructs were introduced into Agrobacterium tumefaciens GV301. Then, VIGS assays were carried out as previously described (Fu et al., 2005).

To generate amiRNA for silencing SlGCN5, the amiRNAs (21-nt) were designed by using the web MicroRNA Designer $\left(\mathrm{WMD}^{2}\right)$. Pre-amiRNA was assembled by several rounds of PCR using primers listed in Supplementary Table 1. The final PCR fragments were driven under $35 \mathrm{~S}$ promoters in $\mathrm{pCHF} 3$ vector. After SlGCN5-RNAi construct is transformed into Agrobacterium GV3101, the Agrobacterium-mediated transformation of tomato cotyledons was performed as described (Cortina and CuliáñezMacià, 2004; Tripodi, 2020).

\section{Phylogenetic Analysis}

For phylogenetic analysis, the coding sequences of ADA2 orthologs were retrieved from JGI Genome Portal and Resources for Plant Comparative Genomics ${ }^{3}$ by BLAST using AtADA2a coding sequence as a query with default parameters. The phylogenetic tree of ADA2 orthologs in dicots was constructed by W-IQ-TREE (Nguyen et al., 2015), which identified the best evolutionary model as the general time reversible model $(\mathrm{GTR}+\mathrm{F}+\mathrm{I}+\mathrm{G} 4)$. The non-parametric UltraFast Bootstrap (UFBoot) method (Minh et al., 2013) was used to calculate the node support, and 1,000 bootstrap pseudo replicates were performed with bootstrap values indicated in branches.

\section{Subcellular Localization Analysis}

DNA fragment of SlGCN5 was amplified by PCR (primers are listed in Supplementary Table 1) and inserted into pGreenII vector to generate the SlGCN5-GFP (green fluorescent protein) fusion protein. Then, pGreenII vector-based 35S:SlGCN5GFP and the control vector pGreenII-based 35S:GFP were transformed into A. tumefaciens strain GV3101 and injected into 4-week-old tobacco leaves. GFP fluorescence was observed using Olympus (BX53) microscope after $72 \mathrm{~h}$ of infiltration.

\section{RNA Extraction and Expression Analyses}

RNA extraction and quantitative real-time (qRT)-PCR analysis were carried out as described previously (Sun et al., 2019).

\footnotetext{
${ }^{1}$ https://solgenomics.net

${ }^{2}$ http://wmd3.weigelworld.org./cgi-bin/webapp.cgi

${ }^{3}$ https://phytozome-next.jgi.doe.gov/
} 

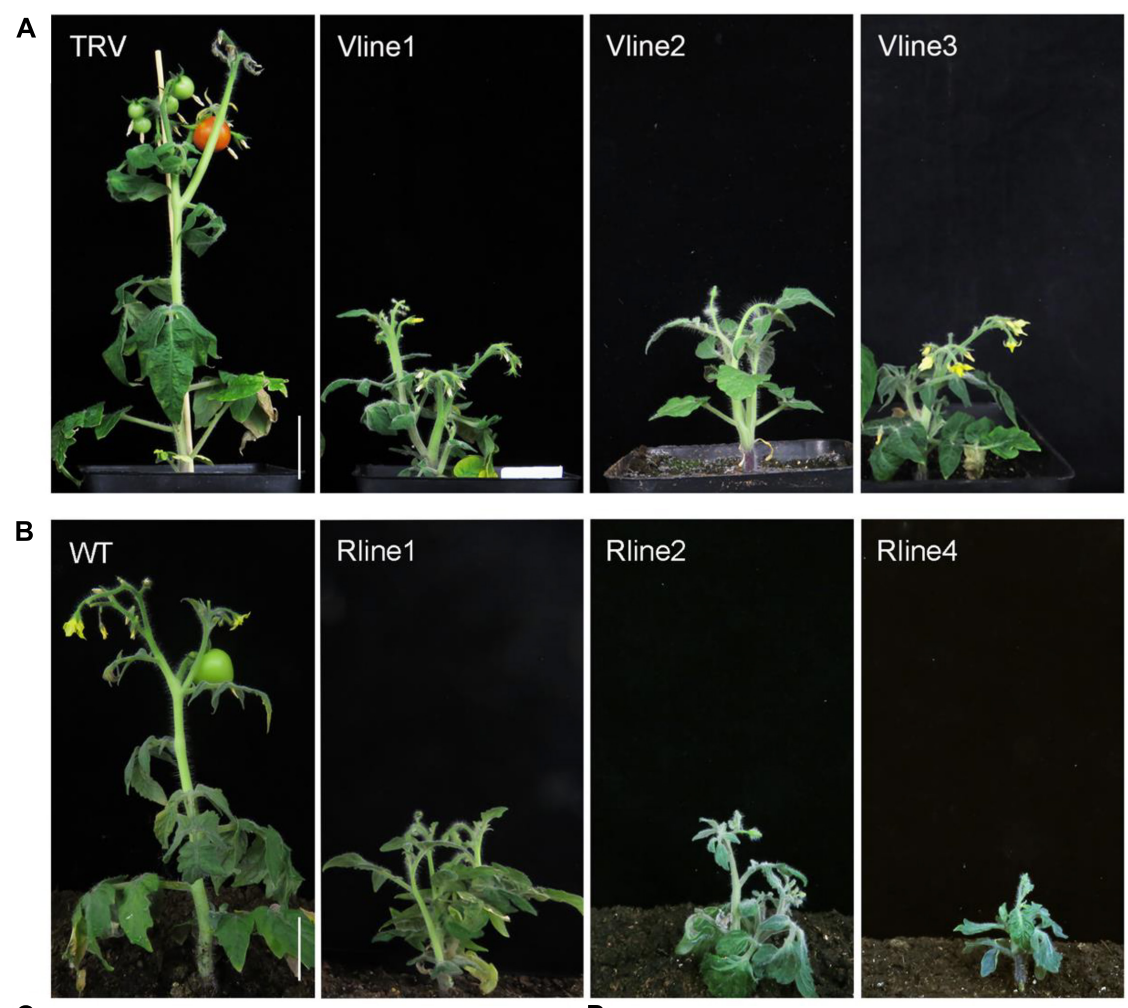

C
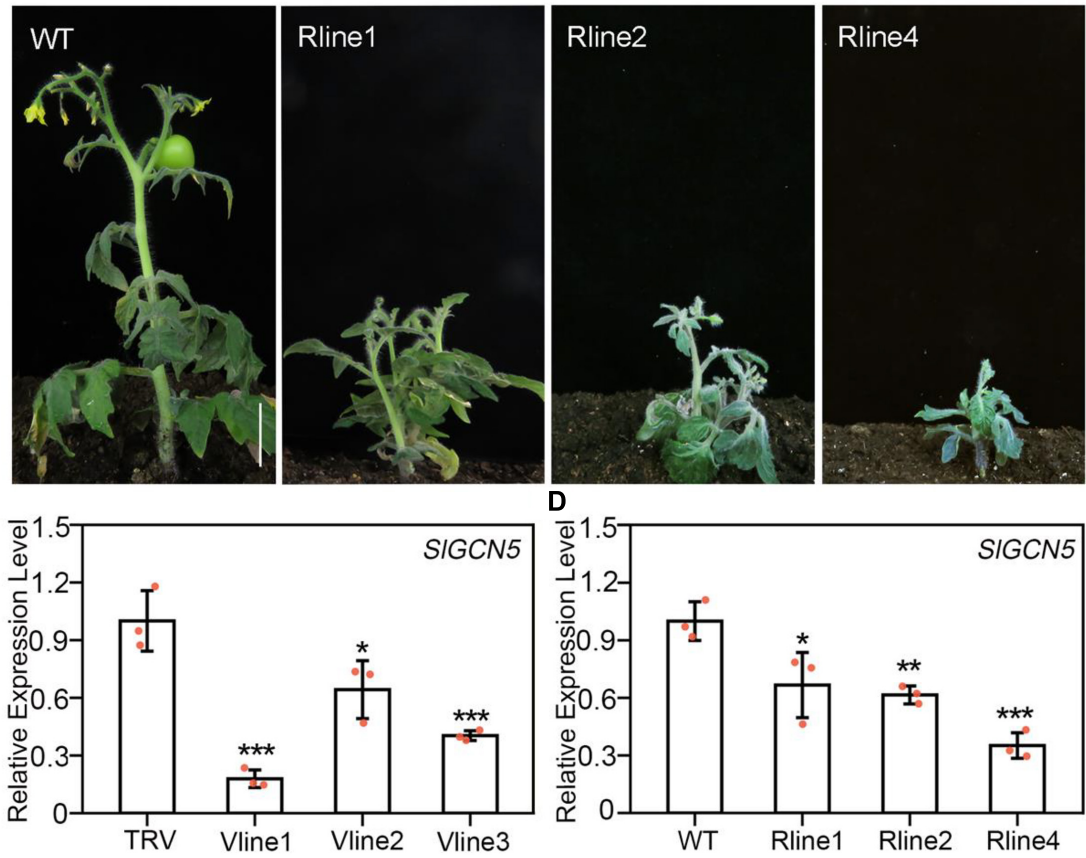

FIGURE 1 | Silencing of SIGCN5 affects plant development. (A) Phenotype of three independent TRV-SIGCN5 lines of Micro-Tom tomato. V lines represent SIGCN5-VIGS lines. (B) Phenotype of three independent SIGCN5-RNAi lines. (C) qRT-PCR analysis of SIGCN5 transcripts in TRV control and TRV-SIGCN5 plants. (D) Transcript levels of S/GCN5 in S/GCN5-RNAi lines relative to wild type (WT). Error bars represent SD of three biological replicates. Asterisks indicate significant differences $\left({ }^{*} p<0.05,{ }^{* *} p<0.01\right.$, and $\left.{ }^{* * *} p<0.001\right)$. Scale bars $=3 \mathrm{~cm}$.

ACTIN2 and SlACTIN2 were served as the internal control in Arabidopsis and tomato, respectively. The sequences of all primers are listed in Supplementary Table 1.

\section{In situ Hybridization}

RNA in situ hybridization was performed as described previously (Sun et al., 2019). Briefly, SlGCN5 (Solyc10g045400.1.1) and SlWUS (Solyc02g083950) probes were synthesized from cDNA by using the primers listed in Supplementary Table 1, and the PCR products were cloned into pGEM-T Easy vector (TIANGEN, VT307). After linearization, the DIG RNA labeling kit (Roche, 11175025910) was used for in vitro transcription of probes. The experiments were performed twice using two different batches of plants. Photographs were taken by using an Olympus BX53 microscope.

\section{Yeast Two-Hybrid Assay}

To obtain yeast two-hybrid vectors, the full-length SlGCN5 was cloned into pGADT7 (Clontech). The full-length SlADA2a and $S I A D A 2 b$ were individually cloned into pGBKT7 (Clontech). The yeast two-hybrid assay was performed using the Yeastmaker Yeast Transformation System 2 (Clontech, T2001) according to the instruction of the manufacturer. Primer sequences are provided in Supplementary Table 1.

\section{Bimolecular Fluorescence \\ Complementation Assay}

For bimolecular fluorescence complementation (BiFC) assay, SlGCN5 and SlADA2 were tagged with the C-terminal part of YFP (YFPC) and the N-terminal part of YFP (YFPN), respectively, as previously described (Kudla and Bock, 2016). 


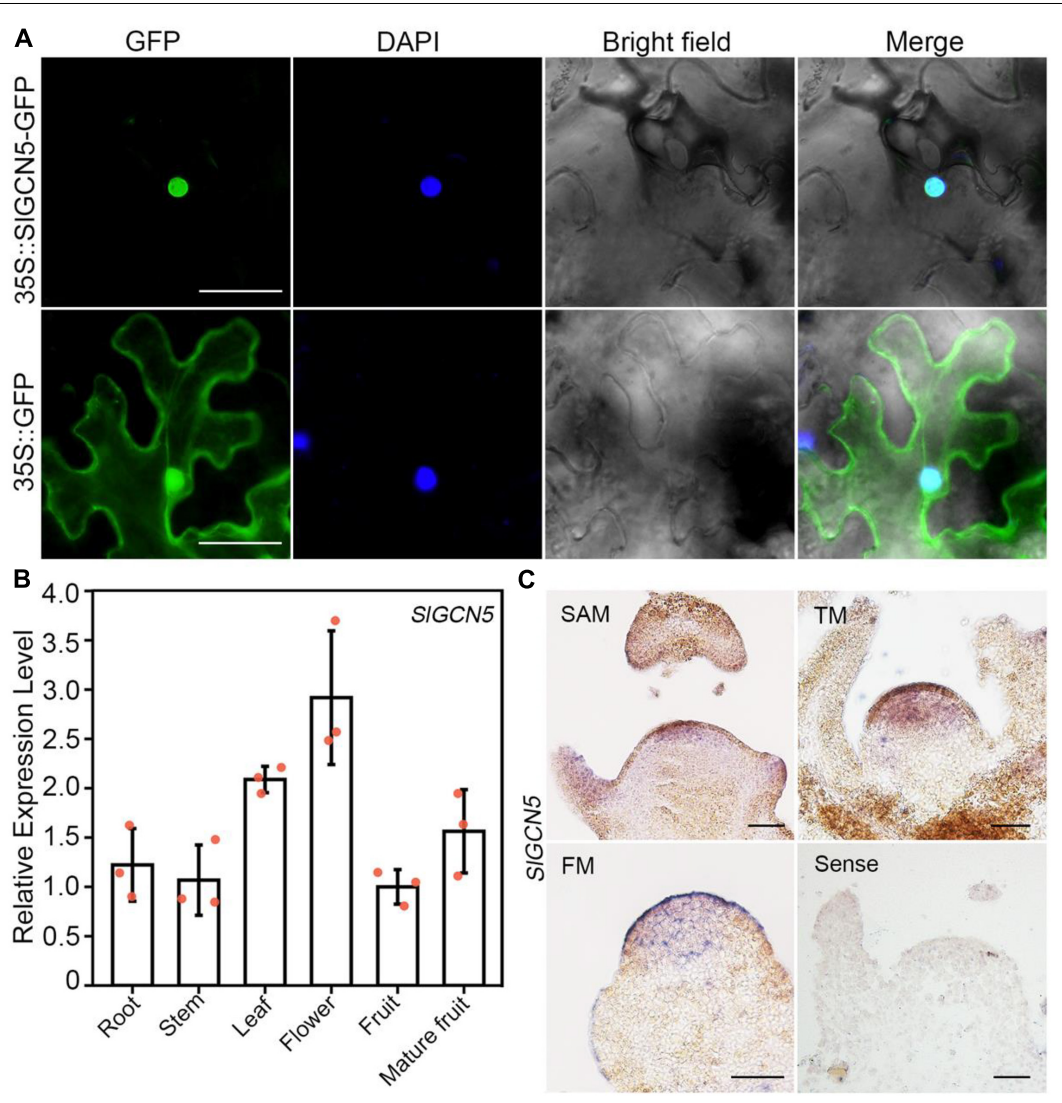

FIGURE 2 | Subcellular localization and gene expression pattern of SIGCN5. (A) Subcellular localization of SIGCN5 in nuclei. 35S:SIGCN5-GFP represents SIGCN5-GFP fusion protein. 35S:GFP represents the control. Scale bars $=20 \mu \mathrm{m}$. (B) qRT-PCR analysis of SIGCN5 in different tomato organs. SIACTIN served as the internal control. Error bars represent SD of three biological replicates. (C) In situ hybridization of S/GCN5 in SAM, floral transition meristem (TM), and FM, respectively. Scale bars $=50 \mu \mathrm{m}$.

Cloning primers are listed in Supplementary Table 1. After vectors were transformed into Agrobacterium, the Agrobacterium carrying different vectors were co-infiltrated into tobacco (Nicotiana benthamiana) leaves of 4-week-old plants as described previously (Sparkes et al., 2006). The infected tobacco leaves were cultured for $72 \mathrm{~h}$ before observation. Notably, $5 \mu \mathrm{g} / \mathrm{ml}$ DAPI was used to visualize the nuclei. The fluorescence was observed by using Olympus (BX53) microscope.

\section{Statistical Analysis}

The statistical analysis was conducted using two-tailed $t$-test. The statistically significant differences are indicated by ${ }^{*} p<0.05$, ** $p<0.01$, or ${ }^{* * *} p<0.001$.

\section{RESULTS}

\section{Silencing of SIGCN5 Affects Tomato Plant Development}

GCN5 was reported to participate in many biological processes in Arabidopsis, especially in plant development (Vlachonasios et al., 2003). In this study, we aimed to investigate the function of GCN5 in tomato development. For this purpose, we first searched for putative homologs of AtGCN5 in tomato genome sequence, and only one homologous gene with three isoforms was identified (Supplementary Figure 1). Among these three isoforms, we chose the one with the highest expression level in tomato inflorescences and the highest protein similarity with AtGCN5 for further study. To explore the effect of SlGCN5 silencing, tomato seedlings in two-cotyledon stage were infected with Agrobacterium carrying the TRV-based VIGS of SlGCN5 vector. TRV-SlGCN5 plants exhibited predominantly developmental defects, including reduced plant height, loss of shoot apical dominance, altered pattern of axillary shoot development, shortened internode, late flowering, and male sterility (Figure 1A), suggesting that SIGCN5 is required in various tomato plant developmental processes. To verify the phenotype of TRV-SlGCN5, we created SlGCN5-RNAi plants and found all three of the SlGCN5-RNAi lines exhibited similar phenotype with TRV-SlGCN5 plants (Figure 1B).

Results of qRT-PCR showed that SlGCN5 transcription level in the TRV-SlGCN5-infected plants was significantly lower than plants infected with TRV control (Figure 1C), confirming that the abnormal phenotypes are caused by SlGCN5 gene silencing. Similarly, the expression level of SlGCN5 was significantly reduced in the RNAi lines compared with WT plants (Figure 1D). 
A

SIGCN5-AD
SIADA2b-BD
SIGCN5-AD
$B D$
$A D$
SIADA2b-BD
$A D$
SIADA2a-BD
SIGCN5-AD
SIADA2a-BD

B

\section{SIGCN5-cYFP SIADA2b-nYFP}

SIGCN5-cYFP
nYFP

$$
\begin{array}{r}
\text { cYFP } \\
\text { SIADA2b-nYFP }
\end{array}
$$

SIGCN5-CYFP SIADA2a-nYFP

$$
\begin{array}{r}
\text { CYFP } \\
\text { SIADA2a-nYFP }
\end{array}
$$

SIADA2a-nYFP
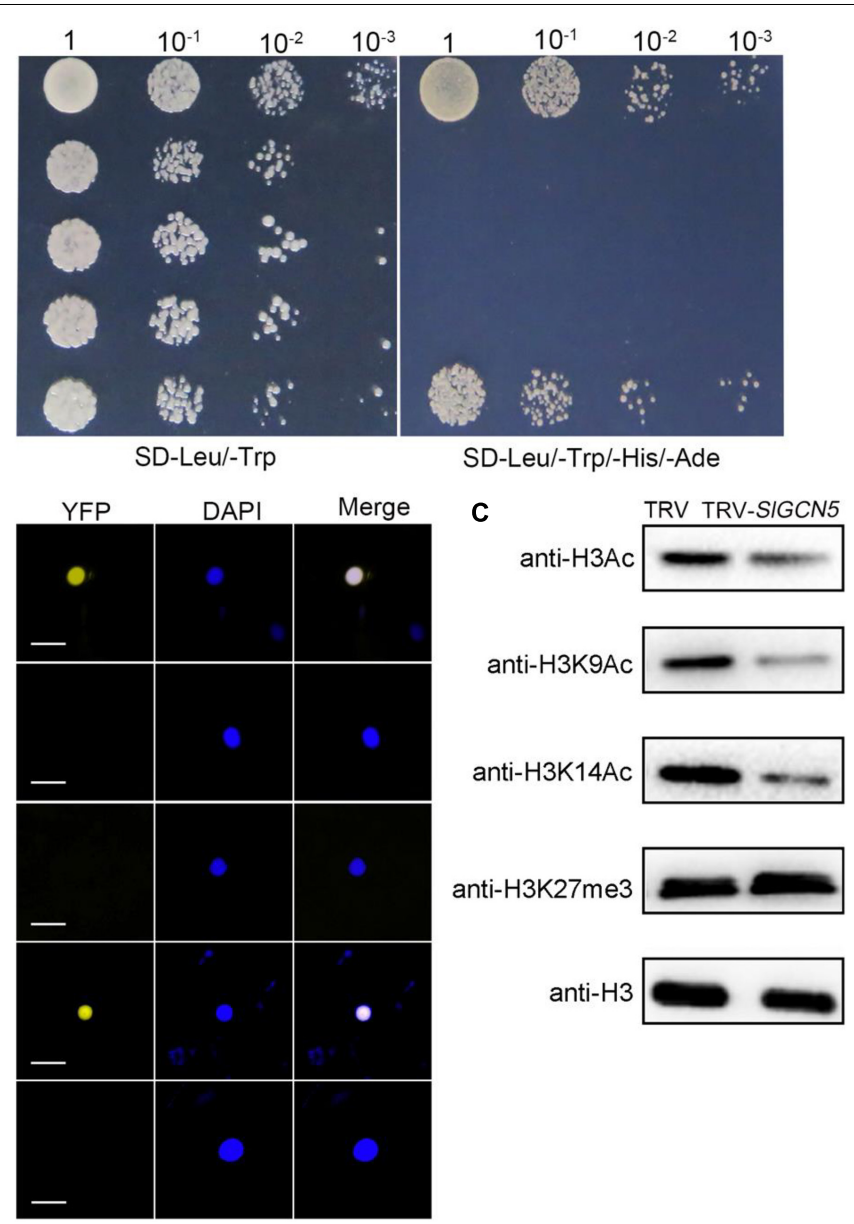

FIGURE 3 | SIGCN5 functions as a histone H3 acetyltransferase. (A) Yeast two-hybrid assays of SIGCN5, SIADA2a, and SIADA2b. Full-length cDNAs of SIGCN5, SIADA2a, and SIADA2b were cloned into AD (the prey plasmid pGADT7) and BD (the bait plasmid pGBKT7), respectively. Yeast cells transformed with the indicated plasmids were grown on medium lacking leucine and tryptophan (SD/-Leu/-Trp) and selective medium lacking leucine, tryptophan, histidine, and adenine (SD/-Leu/-Trp/-His/-Ade). (B) Bimolecular fluorescent complementation analysis in tobacco leaves. Merge refers to merged images for yellow fluorescent protein (YFP) and DAPI fluorescence. SIGCN5 and SIADA2a/b were fused to cCFP and nYFP, respectively. Scale bars $=20 \mu \mathrm{m}$. (C) Histone acetyltransferase activity of SIGCN5 determined by in vivo histone acetyltransferase assay. Histone acetylation levels were detected by immunoblotting with antibodies of the indicated histone acetylation marks in TRV and TRV-SIGCN5 plants. Anti-H3 antibody was used as loading control.

Due to the similar phenotypes of SlGCN5-RNAi and TRVSlGCN5 plants, we used TRV-SlGCN5 plants for subsequent functional studies in tomato plant development.

\section{SIGCN5 Is Located in the Nucleus and Highly Expresses in Tomato Early Floral Bud}

To investigate the expression pattern of SlGCN5, we first analyzed subcellular localization of SIGCN5 protein. Results showed that SIGCN5-GFP fusion protein driven by constitutive cauliflower mosaic virus $35 \mathrm{~S}$ promoter exclusively localized in the nucleus (Figure 2A), suggesting that SlGCN5 may have a putative role in histone modification. During tomato plant development, SlGCN5 transcripts expressed widely in roots, stems, leaves, flowers, and fruits (Figure 2B). Our in situ hybridization assays revealed that SlGCN5 is strongly expressed in the upper cell layers of SAM. Meanwhile, SlGCN5 was expressed throughout the entire floral transition meristem and FM of WT plants, which may overlap with the expression domain of SlWUS (Figure 2C), hinting at a potential role for SIGCN5 in regulation of meristematic activities.

\section{SIGCN5 Catalyzes Histone Acetylation}

The SAGA (Spt-Ada-Gcn5 acetyltransferase) complex is highly conserved for active regulation of gene transcription in yeast and plants (Carrozza et al., 2003; Vlachonasios et al., 2003; Zhou et al., 2017; Li et al., 2019). We also identified ADA2aand ADA2b-like proteins in tomato (Supplementary Figure 2) and named them as SIADA2a and SIADA2b, respectively, which have the highest homology with AtADA2a and AtADA2b in Arabidopsis. SlADA2a and SlADA2b have 3 and 2 isoforms respectively. According to the transcript analysis results in tomato inflorescences, XP_004243566 and XP_004239816 were selected as representatives of SIADA2 $a$ and SIADA2 $b$ for further study (Supplementary Figure 3). To confirm the interactions 

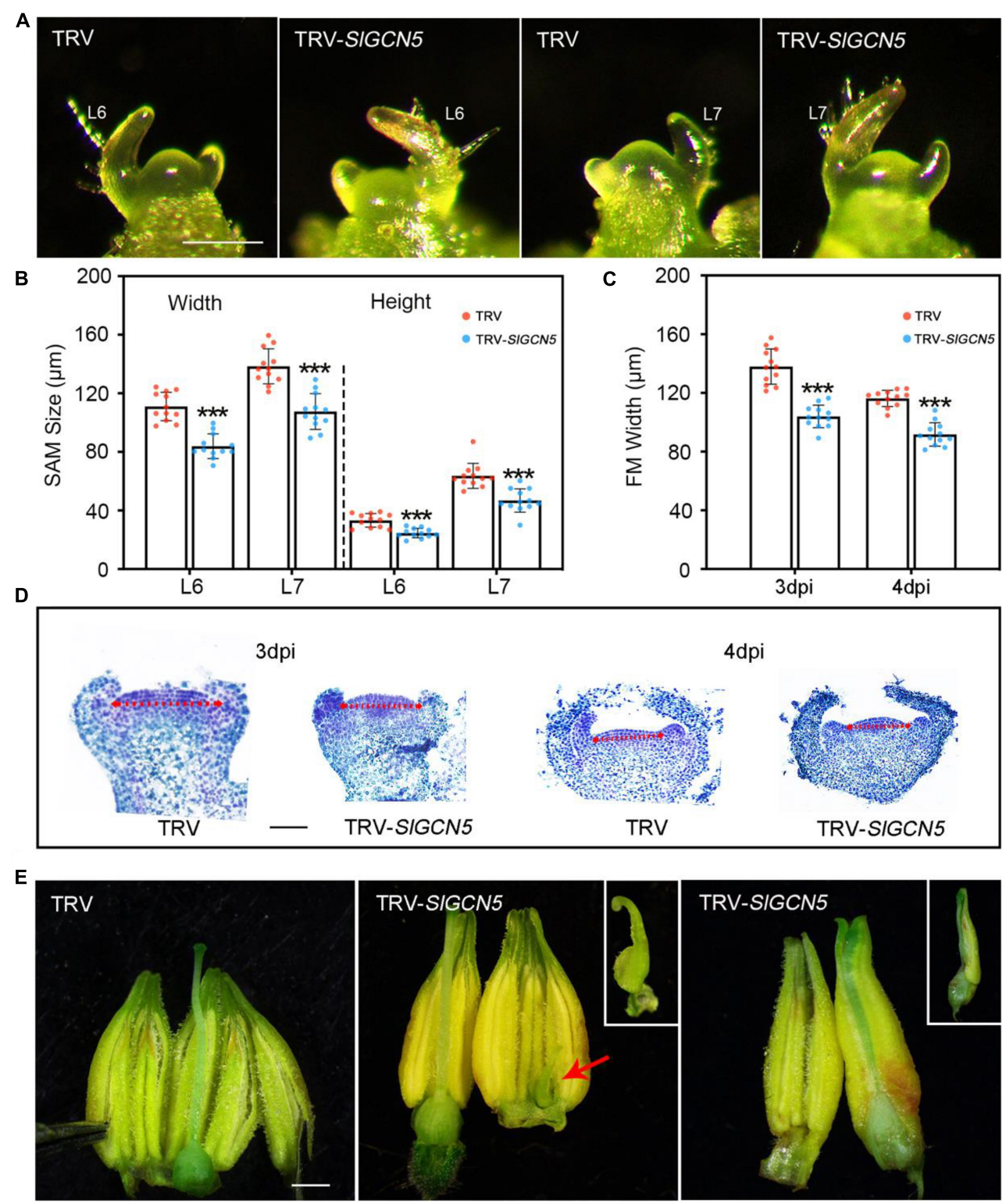

FIGURE 4 | SAM and floral phenotype of silenced-S/GCN5 plants. (A) Images of the SAMs from TRV control and TRV-S/GCN5 plants. L6 and L7 indicate Leaf 6 and Leaf 7, respectively. Scale bars $=200 \mu \mathrm{m}$. (B) SAM size from TRV control and TRV-S/GCN5 plants. Error bar indicates SD of 12 biological replicates. (C) FM size from TRV control and TRV-SIGCN5 plants at 3 and 4 dpi (days post floral initiation). Error bar indicates SD of 12 biological replicates. (D) Longitudinal sections of floral meristem of TRV control and TRV-S/GCN5 plants. The red dash arrow marks the width of each floral meristem. Scale bars $=50 \mu \mathrm{m}$. (E) Flowers of TRV and TRV-SIGCN5. Scale bars $=1 \mathrm{~mm}$. Asterisks indicate significant differences between TRV control and TRV-SIGCN5 $\left.{ }^{(\star \star} p<0.001\right)$.

between SlADA2a with SIGCN5 and SlADA2b with SlGCN5, we cloned the full-length cDNAs of SlADA2a, SlADA2b, and SlGCN5 and performed yeast two-hybrid assays. The results showed that SIGCN5 can interact with both SIADA2a and SlADA2b in yeast cells (Figure 3A). To verify the yeast twohybrid results, we performed BiFC analysis in tobacco (Nicotiana tabacum) leaves. SIGCN5 was fused to the C-terminus of YFP and named as SIGCN5-cYFP. SIADA2a or SIADA2b was fused to the $\mathrm{N}$-terminus of YFP and named as SlADA2a-nYFP or SlADA2bnYFP, respectively. We noticed interactions between SIGCN5 and
SlADA2a, as well as SIGCN5 and SlADA2b in the nucleus, both of which gave clear signals (Figure 3B). These results suggest that SlGCN5 can interact with both SIADA2a and SlADA2b and that the three proteins may form a protein complex.

To test the HAT activity of SlGCN5 in vivo, we compared histone acetylation levels in TRV-SlGCN5 plants with TRV control plants by immunoblotting, using anti-H3K9Ac, anti-H3K14Ac, anti-H3Ac, and anti-H3K27me3 antibodies. Our results revealed that obvious reduction of $\mathrm{H} 3 \mathrm{ac}$, H3K9ac, and H3K14ac levels in TRV-SlGCN5 compared with 

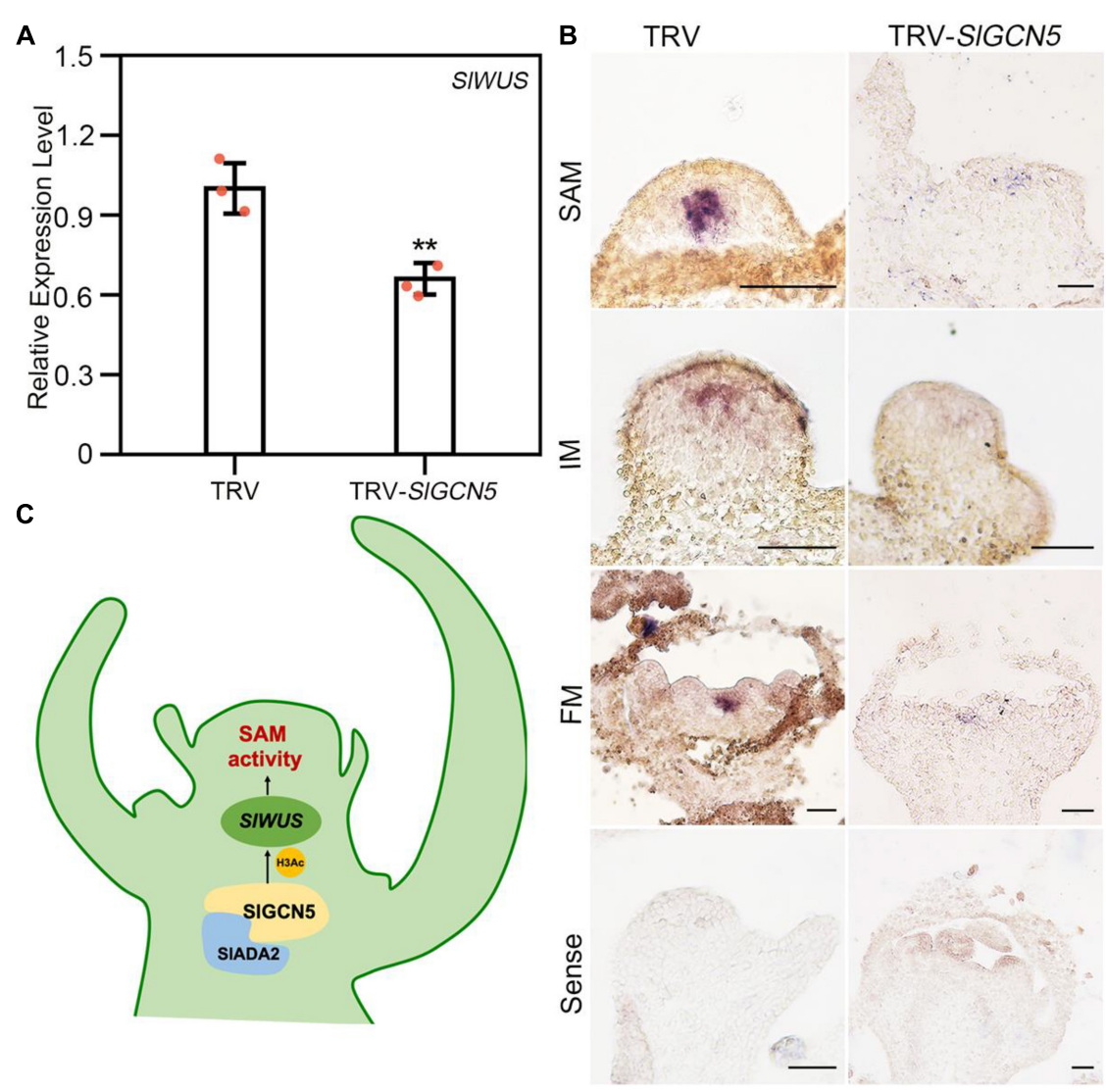

FIGURE 5 | SIGCN5 positively regulates SIWUS in tomato. (A) qRT-PCR analysis of SIWUS expression level in SAM. The error bar represents SD of three biological replicates. The asterisks indicate significant differences between TRV and TRV-SIGCN5 (** $p<0.01)$. (B) Expression of SIWUS mRNA in TRV control and TRV-SIGCN5 plants by in situ hybridization. Scale bars $=50 \mu \mathrm{m}$. (C) Model: SIGCN5 together with SIADA2a and SIADA2b could form HAT complex, which positively regulate SIWUS to ensure the proper development of SAM.

the TRV control plants (Figure 3C), suggesting that SlGCN5 can catalyze acetylation on histone $\mathrm{H} 3$, specifically at $\mathrm{H} 3 \mathrm{~K} 9$ and $\mathrm{H} 3 \mathrm{~K} 14$ residues. These results are consistent with the known function of AtGCN5, which was reported to catalyze H3K14ac, and additional histone residues, including $\mathrm{H} 3 \mathrm{~K} 9, \mathrm{H} 3 \mathrm{~K} 18$, $\mathrm{H} 3 \mathrm{~K} 27$, and $\mathrm{H} 3 \mathrm{~K} 36$, and other histones such as $\mathrm{H} 4$ and $\mathrm{H} 2 \mathrm{~B}$ in Arabidopsis (Kuo et al., 1996; Grant et al., 1997; Morris et al., 2007). To confirm the role of SlGCN5 in plant development, we generated transgenic Arabidopsis plants by transforming the null-mutant gcn5-7 with 35S:SlGCN5-GFP. 35S:SlGCN5-GFP gcn5-7 plants have noticeable gene and protein expressions of SlGCN5, which are examined by qRT-PCR and Western blot (Supplementary Figure 4A). Furthermore, 35S:SlGCN5-GFP gcn5-7 plants show almost fully rescued phenotype compared with $g c n 5-7$ (Supplementary Figure 4B), indicating that SIGCN5 functions similarly as AtGCN5.

\section{SIGCN5 Regulates Tomato Shoot Meristem and Flower Development}

SlGCN5-silenced plants exhibited reduced plant height. Thus, we measured SAM size in TRV-SlGCN5 and TRV control plants and observed reduced SAM size in TRV-SlGCN5 at different developmental stages compared with TRV control plants (Figures 4A,B). We also observed reduced FM width but relatively unchanged FM height (Supplementary Figure 5) in TRV-SlGCN5 young floral buds prior to the emergence of the carpel primordia (Figures 4C,D). Although FM size in TRV-SlGCN5 is reduced, floral organ number remains largely unaffected. However, in TRV-SlGCN5 flowers, we occasionally noticed some carpelloid stamens and carpels fused with stamens [2/15 (13.3\%) independent transgenic lines show abnormal flowers] (Figure 4E). These results implied that silencing of SlGCN5 resulted in reduced SAM and FM sizes in tomato and may also influence reproductive floral organ development.

\section{SIGCN5 Positively Regulates SIWUS Expression}

The reduced SAM and FM size leads us to examine expression changes of SlWUS in TRV-SlGCN5 plants. Expression analysis by qRT-PCR revealed that SlWUS transcript level was significantly reduced in TRV-SlGCN5 meristems (Figure 5A). To validate the qRT-PCR results, expression pattern of SlWUS was examined by in situ hybridization assays. We noticed obviously reduced expression of SlWUS mRNA in TRV-SlGCN5 SAMs and FMs 
(Figure 5B) compared with TRV control plants. These results suggested that SIGCN5 may positively regulate SIWUS expression in tomato shoot meristem and FM. Furthermore, we observed remarkable decrease in the transcript level of SlCLV1 and $S l C L V 3$, the other two key factors in $C L V$-WUS feedback loop, in TRV-SlGCN5 meristems by qRT-PCR analysis (Supplementary Figure 6). These results indicate that SlGCN5 may potentially regulate multiple genes in meristem development of tomato.

\section{DISCUSSION}

Histone lysine acetylation is an essential chromatin modification for epigenetic regulation of gene expression in plant development and plant response to environmental stress. AtGCN5 was identified as the first transcription-linked HAT (Brownell et al., 1996), with specificity for histone H3K14ac (Kuo et al., 1996). In addition, GCN5 could also acetylate histone lysine residues such as $\mathrm{H} 3 \mathrm{~K} 9, \mathrm{H} 3 \mathrm{~K} 18, \mathrm{H} 3 \mathrm{~K} 23, \mathrm{H} 3 \mathrm{~K} 27$, and H3K36 and other histones such as H4 and H2B (Grant et al., 1997; Morris et al., 2007). The SAGA complex is an evolutionarily conserved HAT complex (Spedale et al., 2012), which catalyzes histone acetylation for modulating gene expression and participates in various developmental processes in eukaryotes. In this study, we showed that SIGCN5 can acetylate histones $\mathrm{H} 3 \mathrm{~K} 9$ and $\mathrm{H} 3 \mathrm{~K} 14$ at the genomic level in tomato, and SlGCN5 also interacts with SIADA2a and SlADA2b to form HAT unit.

Shoot apical meristem is an organized structure and responds to different development signals. The stem cell pool is maintained within the central zone of the SAM (Fletcher, 2018). Compromised SAM activity leads to premature plant growth stagnation before forming full organs (Laux et al., 1996; Kieffer et al., 2006), whereas plants with overproliferated stem cells in SAM can produce many extra organs (Clark et al., 1993; TaguchiShiobara et al., 2001; Yuste-Lisbona et al., 2020). Therefore, the maintenance of SAM homeostasis is key for plant development. It is well-understood that conserved $C L V$-WUS feedback signaling is important for the maintenance of SAM activity (Somssich et al., 2016), but it is not well-known how this feedback loop is modified in various plant species. In this study, we characterized the function of SIGCN5 and studied its role in SAM maintenance. Our data indicate that SlGCN5 is important to maintain SAM activity in tomato. Weakened SlGCN5 activity affects SAM development and resulted in reduced SAM and FM size (Figure 4). Consistent with the phenotype, we also observed reduced SlWUS expression (Figure 5B) in SAM and FM in the plants with compromised SlGCN5 activity. However, we did not observe obvious changes in floral organ numbers. Instead, we occasionally observed some carpelloid stamens and carpels fused with stamens (Figure 4E). These phenotypes resemble the S. lycopersicum GT11 (SlGT11) mutant, in which the function of floral B-class genes was affected (Yang et al., 2020). Therefore, we suspect that the transformation of floral homeotic genes may also exist in TRV-SlGCN5 plants and that SlGCN5 could participate in the maintenance of floral organ identity.
Modulation of $C L V$-WUS pathway is one important approach to increase crop yield (Fletcher, 2018). In tomato, several transcription factors that could influence the CLV-WUS loop also have been discovered. DEFECTIVE TOMATO MERISTEM (DTM) forms a negative feedback loop with the class III homeodomain-leucine zipper (HD-ZIP III) transcription factors to confine SlCLV3 and SlWUS expression to specific domains in the shoot meristem of tomato (Xu et al., 2019). APETALA2/ethylene responsive factor (AP2/ERF) superfamily transcription factor excessive number of floral organs (ENO) regulates SlWUS expression to restrict stem cell proliferation, thereby maintaining floral stem cell homeostasis (Yuste-Lisbona et al., 2020). In addition to transcription factors, SlWUS expression can also be regulated by chromatin remodeling factors such as histone deacetylase 19 in tomato (Bollier et al., 2018).

In this study, we identified and investigated the function of SlGCN5 in tomato meristem development and found that SlGCN5 acts as an acetyltransferase to activate the expression of SlWUS, thus maintaining SAM activity (Figure 5C). We also noticed SIGCN5 may play a role in floral organ development. These findings could potentially shed light on genetic enhancement of tomato plants.

\section{DATA AVAILABILITY STATEMENT}

The original contributions presented in the study are included in the article/Supplementary Material, further inquiries can be directed to the corresponding author/s.

\section{AUTHOR CONTRIBUTIONS}

BS conceived and designed research, wrote the manuscript, and revised the manuscript. AH and SX conducted experiments. ZY performed data analysis. All authors have read and approved the manuscript.

\section{FUNDING}

This work was supported by the Fundamental Research Funds for the Central Universities (0208/14380167) to BS.

\section{ACKNOWLEDGMENTS}

We would like to thank Zhuqing Shao (Nanjing University) for providing tomato seeds and Bote Luo from Xiaofeng Wang lab (Northwest A\&F University) for providing pTRV1 and PTRV2 vectors.

\section{SUPPLEMENTARY MATERIAL}

The Supplementary Material for this article can be found online at: https://www.frontiersin.org/articles/10.3389/fpls.2021. 805879/full\#supplementary-material 


\section{REFERENCES}

Anzola, J. M., Sieberer, T., Ortbauer, M., Butt, H., Korbei, B., Weinhofer, I., et al. (2010). putative arabidopsis transcriptional adaptor protein (PROPORZ1) is required to modulate histone acetylation in response to auxin. Proc. Natl. Acad. Sci. U.S.A. 107, 10308-10313. doi: 10.1073/pnas.0913918107

Bannister, A. J., and Kouzarides, T. (2011). Regulation of chromatin by histone modifications. Cell Res. 21, 381-395. doi: 10.1038/cr.2011.22

Benhamed, M., Bertrand, C., Servet, C., and Zhou, D.-X. (2006). Arabidopsis GCN5, HD1, and TAF1/HAF2 interact to regulate histone acetylation required for light-responsive gene expression. Plant Cell 18, 2893-2903. doi: 10.1105/tpc. 106.043489

Bertrand, C., Bergounioux, C., Domenichini, S., Delarue, M., and Zhou, D.X. (2003). Arabidopsis histone acetyltransferase AtGCN5 regulates the floral meristem activity through the WUSCHEL/AGAMOUS pathway. J. Biol. Chem. 278, 28246-28251. doi: 10.1074/jbc.M302787200

Bollier, N., Sicard, A., Leblond, J., Latrasse, D., Gonzalez, N., Gevaudant, F., et al. (2018). At-MINI ZINC FINGER2 and Sl-INHIBITOR OF MERISTEM ACTIVITY, a conserved missing link in the regulation of floral meristem termination in Arabidopsis and tomato. Plant Cell 30, 83-100. doi: 10.1105/tpc. 17.00653

Brownell, J. E., Zhou, J., Ranalli, T., Kobayashi, R., Edmondson, D. G., Roth, S. Y., et al. (1996). Tetrahymena histone acetyltransferase A: a homolog to Yeast Gcn5p linking histone acetylation to gene activation. Cell 84, 843-851. doi: 10.1016/S0092-8674(00)81063-6

Carrozza, M. J., Utley, R. T., Workman, J. L., and Côté, J. (2003). The diverse functions of histone acetyltransferase complexes. Trends Genet. 19, 321-329. doi: 10.1016/S0168-9525(03)00115-X

Chu, Y. H., Jang, J. C., Huang, Z., and van der Knaap, E. (2019). Tomato locule number and fruit size controlled by natural alleles of lc and fas. Plant Direct 3:e00142. doi: 10.1002/pld3.142

Clark, S. E., Running, M. P., and Meyerowitz, E. M. (1993). Clavata1, a regulator of meristem and flower development in Arabidopsis. Development 119, 397-418.

Cohen, R., Schocken, J., Kaldis, A., Vlachonasios, K. E., Hark, A. T., and McCain, E. R. (2009). The histone acetyltransferase GCN5 affects the inflorescence meristem and stamen development in Arabidopsis. Planta 230, 1207-1221. doi: 10.1007/s00425-009-1012-5

Cortina, C., and Culiáñez-Macià, F. A. (2004). Tomato transformation and transgenic plant production. Plant Cell Tissue Organ Cult. 76, 269-275. doi: 10.1023/B:TICU.0000009249.14051.77

Daum, G., Medzihradszky, A., Suzaki, T., and Lohmann, J. U. (2014). A mechanistic framework for noncell autonomous stem cell induction in Arabidopsis. Proc. Natl. Acad. Sci. U.S.A. 111:14619.

Fletcher, J. C. (2018). The CLV-WUS stem cell signaling pathway: a roadmap to crop yield optimization. Plants 7:87. doi: 10.3390/plants7040087

Fu, D.-Q., Zhu, B.-Z., Zhu, H.-L., Jiang, W.-B., and Luo, Y.-B. (2005). Virusinduced gene silencing in tomato fruit: VIGS in tomato fruit. Plant J. 43, 299-308. doi: 10.1111/j.1365-313X.2005.02441.x

Galli, M., and Gallavotti, A. (2016). Expanding the regulatory network for meristem size in plants. Trends Genet. 32, 372-383. doi: 10.1016/j.tig.2016.04.001

Grant, P. A., Duggan, L., Cote, J., Roberts, S. M., Brownell, J. E., Candau, R., et al. (1997). Yeast Gcn5 functions in two multisubunit complexes to acetylate nucleosomal histones: characterization of an Ada complex and the SAGA (Spt/Ada) complex. Genes Dev. 11, 1640-1650. doi: 10.1101/gad.11.13.1640

Han, H., Liu, X., and Zhou, Y. (2020). Transcriptional circuits in control of shoot stem cell homeostasis. Curr. Opin. Plant Biol. 53, 50-56.

Kieffer, M., Stern, Y., Cook, H., Clerici, E., Maulbetsch, C., Laux, T., et al. (2006). Analysis of the transcription factor WUSCHEL and its functional homologue in antirrhinum reveals a potential mechanism for their roles in meristem maintenance. Plant Cell 18, 560-573. doi: 10.1105/tpc.105.039107

Kornet, N., and Scheres, B. (2009). Members of the GCN5 histone acetyltransferase complex regulate PLETHORA-mediated root stem cell niche maintenance and transit amplifying cell proliferation in Arabidopsis. Plant Cell 21, 1070-1079. doi: 10.1105/tpc. 108.065300

Kudla, J., and Bock, R. (2016). Lighting the way to protein-protein interactions: recommendations on best practices for bimolecular fluorescence complementation analyses[OPEN]. Plant Cell 28, 1002-1008. doi: $10.1105 /$ tpc. 16.00043
Kuo, M.-H., Brownell, J. E., Sobel, R. E., Ranalli, T. A., Cook, R. G., Edmondson, D. G., et al. (1996). Transcription-linked acetylation by Gcn5p of histones H3 and $\mathrm{H} 4$ at specific lysines. Nature 383, 269-272. doi: 10.1038/383269a0

Laux, T., Mayer, K. F. X., Berger, J., and Jurgens, G. (1996). The WUSCHEL gene is required for shoot and floral meristem integrity in Arabidopsis. Development $122,87-96$.

Li, H., Qi, M., Sun, M., Liu, Y., Liu, Y., Xu, T., et al. (2017). Tomato Transcription factor SIWUS plays an important role in tomato flower and locule development. Front. Plant Sci. 8:457. doi: 10.3389/fpls.2017.00457

Li, S., Lin, Y.-C. J., Wang, P., Zhang, B., Li, M., Chen, S., et al. (2019). The AREB1 transcription factor influences histone acetylation to regulate drought responses and tolerance in populus trichocarpa. Plant Cell 31, 663-686. doi: 10.1105/tpc.18.00437

Mao, Y., Pavangadkar, K. A., Thomashow, M. F., and Triezenberg, S. J. (2006). Physical and functional interactions of Arabidopsis ADA2 transcriptional coactivator proteins with the acetyltransferase GCN5 and with the cold-induced transcription factor CBF1. Biochim. Biophys. Acta 1759, 69-79.

Minh, B. Q., Nguyen, M. A., and von Haeseler, A. (2013). Ultrafast approximation for phylogenetic bootstrap. Mol. Biol. Evol. 30, 1188-1195. doi: 10.1093/molbev/ mst024

Morris, S. A., Rao, B., Garcia, B. A., Hake, S. B., Diaz, R. L., Shabanowitz, J., et al. (2007). Identification of histone $\mathrm{H} 3$ lysine 36 acetylation as a highly conserved histone modification. J. Biol. Chem. 282, 7632-7640. doi: 10.1074/ jbc.M607909200

Muños, S., Ranc, N., Botton, E., Bérard, A., Rolland, S., Duffé, P., et al. (2011). Increase in tomato locule number is controlled by two single-nucleotide polymorphisms located near WUSCHEL1[C][W]. Plant Physiol. 156, 22442254. doi: 10.1104/pp.111.173997

Nguyen, L.-T., Schmidt, H. A., von Haeseler, A., and Minh, B. Q. (2015). IQ-TREE: a fast and effective stochastic algorithm for estimating maximum-likelihood phylogenies. Mol. Biol. Evol. 32, 268-274. doi: 10.1093/molbev/msu300

Périlleux, C., Lobet, G., and Tocquin, P. (2014). Inflorescence development in tomato: gene functions within a zigzag model. Front. Plant Sci. 5:121. doi: 10.3389/fpls.2014.00121

Pfeiffer, A., Wenzl, C., and Lohmann, J. U. (2017). Beyond flexibility: controlling stem cells in an ever changing environment. Curr. Opin. Plant Biol. 35, 117-123. doi: 10.1016/j.pbi.2016.11.014

Rodríguez-Leal, D., Lemmon, Z. H., Man, J., Bartlett, M. E., and Lippman, Z. B. (2017). Engineering quantitative trait variation for crop improvement by genome editing. Cell 171, 470-480.e8. doi: 10.1016/j.cell.2017.08.030

Ruggieri, V., Alexiou, K., Morata, J., Argyris, J., and Benhamed, M. (2020). GCN5 modulates salicylic acid homeostasis by regulating H3K14ac levels at the $5^{\prime}$ and $3^{\prime}$ ends of its target genes. Nucleic Acids Res. 48, 5953-5966.

Schmitz, G., and Theres, K. (1999). Genetic control of branching in Arabidopsis and tomato. Curr. Opin. Plant Biol. 2, 51-55. doi: 10.1016/S1369-5266(99)80010-7

Schoof, H., Lenhard, M., Haecker, A., Mayer, K. F. X., Jürgens, G., and Laux, T. (2000). The stem cell population of arabidopsis shoot meristems is maintained by a regulatory loop between the CLAVATA and WUSCHEL genes. Cell 100, 635-644. doi: 10.1016/S0092-8674(00)80700-X

Sekhar, K., and Sawhney, V. K. (1984). A scanning electron microscope study of the development and surface features of floral organs of tomato (Lycopersicon esculentum). Can. J. Bot. 62, 2403-2413.

Servet, C., Conde e Silva, N., and Zhou, D.-X. (2010). Histone acetyltransferase AtGCN5/HAG1 is a versatile regulator of developmental and inducible gene expression in Arabidopsis. Mol. Plant 3, 670-677. doi: 10.1093/mp/ssq018

Shahbazian, M. D., and Grunstein, M. (2007). Functions of site-specific histone acetylation and deacetylation. Annu. Rev. Biochem. 76, 75-100. doi: 10.1146/ annurev.biochem.76.052705.162114

Shang, E., Ito, T., and Sun, B. (2019). Control of floral stem cell activity in Arabidopsis. Plant Signal. Behav. 14:1659706. doi: 10.1080/15592324.2019. 1659706

Somssich, M., Je, B. I., Simon, R., and Jackson, D. (2016). CLAVATA-WUSCHEL signaling in the shoot meristem. Development 143, 3238-3248. doi: 10.1242/ dev. 133645

Sparkes, I. A., Runions, J., Kearns, A., and Hawes, C. (2006). Rapid, transient expression of fluorescent fusion proteins in tobacco plants and generation of stably transformed plants. Nat. Protoc. 1, 2019-2025. doi: 10.1038/nprot.2006. 286 
Spedale, G., Timmers, H. T. M., and Pijnappel, W. W. M. P. (2012). ATACking the complexity of SAGA during evolution. Genes Dev. 26, 527-541. doi: 10.1101/gad.184705.111

Sun, B., Zhou, Y., Cai, J., Shang, E., Yamaguchi, N., Xiao, J., et al. (2019). Integration of transcriptional repression and polycomb-mediated silencing of WUSCHEL in floral meristems. Plant Cell 31, 1488-1505. doi: 10.1105/tpc.18.0 0450

Taguchi-Shiobara, F., Yuan, Z., Hake, S., and Jackson, D. (2001). The fasciated ear2 gene encodes a leucine-rich repeat receptor-like protein that regulates shoot meristem proliferation in maize. Genes Dev 15, 2755-2766. doi: 10.1101/gad. 208501

Tripodi, P. (2020). Crop Breeding: Genetic Improvement Methods. Methods in Molecular Biology. New York, NY: Humana.

Vlachonasios, K. E., Thomashow, M. F., and Triezenberg, S. J. (2003). Disruption mutations of ADA2b and GCN5 transcriptional adaptor genes dramatically affect arabidopsis growth, development, and gene expression. Plant Cell 15, 626-638. doi: 10.1105/tpc.00 7922

Xu, C., Liberatore, K. L., MacAlister, C. A., Huang, Z., Chu, Y.-H., Jiang, K., et al. (2015). A cascade of arabinosyltransferases controls shoot meristem size in tomato. Nat. Genet. 47, 784-792. doi: 10.1038/ng.3309

Xu, Q., Li, R., Weng, L., Sun, Y., Li, M., and Xiao, H. (2019). Domainspecific expression of meristematic genes is defined by the LITTLE ZIPPER protein DTM in tomato. Commun. Biol. 2:134. doi: 10.1038/s42003-019-0 368-8

Yadav, R. K., Perales, M., Gruel, J., Girke, T., Jnsson, H., and Reddy, G. V. (2011). WUSCHEL protein movement mediates stem cell homeostasis in the Arabidopsis shoot apex. Genes Dev. 25, 2025-2030.
Yang, L., Qi, S., Touqeer, A., Li, H., Zhang, X., Liu, X., et al. (2020). SlGT11 controls floral organ patterning and floral determinacy in tomato. BMC Plant Biol. 20:562. doi: 10.1186/s12870-020-02760-2

Yuste-Lisbona, F. J., Fernández-Lozano, A., Pineda, B., Bretones, S., Ortíz-Atienza, A., García-Sogo, B., et al. (2020). ENO regulates tomato fruit size through the floral meristem development network. Proc. Natl. Acad. Sci. U.S.A. 117, 8187-8195. doi: 10.1073/pnas.1913688117

Zhou, S., Jiang, W., Long, F., Cheng, S., Yang, W., Zhao, Y., et al. (2017). Rice homeodomain protein WOX11 recruits a histone acetyltransferase complex to establish programs of cell proliferation of crown root meristem. Plant Cell 29, 1088-1104. doi: 10.1105/tpc.16.00908

Conflict of Interest: The authors declare that the research was conducted in the absence of any commercial or financial relationships that could be construed as a potential conflict of interest.

Publisher's Note: All claims expressed in this article are solely those of the authors and do not necessarily represent those of their affiliated organizations, or those of the publisher, the editors and the reviewers. Any product that may be evaluated in this article, or claim that may be made by its manufacturer, is not guaranteed or endorsed by the publisher.

Copyright (c) 2022 Hawar, Xiong, Yang and Sun. This is an open-access article distributed under the terms of the Creative Commons Attribution License (CC BY). The use, distribution or reproduction in other forums is permitted, provided the original author(s) and the copyright owner(s) are credited and that the original publication in this journal is cited, in accordance with accepted academic practice. No use, distribution or reproduction is permitted which does not comply with these terms. 